\title{
Atrial Fibrillation Detection Using Feature Based Algorithm and Deep Convolutional Neural Network
}

\author{
Shadi Ghiasi, Mostafa Abdollahpur, Nasimalsadat Madani, Kamran Kiani, Ali Ghaffari \\ Cardiovascular Research Group (CVRG), Department of Mechanical Engineering at K. N. Toosi \\ University of Technology, Tehran, Iran
}

\begin{abstract}
Aims: Electrocardiographic waveforms (ECG) are recognized as the most reliable method to detect abnormal heart rhythms such as atrial fibrillation. This task is challenging when the signals are distorted by noise. This paper presents an automatic classification algorithm to classify short lead ECGs in terms of abnormality of heart rhythm (AF or alternative rhythms) and quality (noisy recordings).

Methods: To meet this end, at first baseline wander removal and Butterworth filter for each signal are applied as a preprocessing stage. Due to the existence of noise in recordings, high quality beats are selected for any further analysis using cycle quality assessment. Then, three sets of features defined as correlation coefficient, fractal dimension and variance of $R$ peaks are extracted to predict noisy recordings. Two separate approaches are employed to classify other three classes. The first approach is the feature based methodology and the second one is the applying deep neural networks. In the first approach, features from different domains are extracted. The method for AF detection utilizes and characterizes variability in RR-intervals which are extracted by applying classic PanTompkins algorithm. To improve the accuracy of the AFdetection, atrial activity is analyzed by understanding whether the P-wave is present in signal. This is done by investigating the morphology of $P$-waves. Heart rate abnormality and the existence of premature beats in a signal are regarded as two characteristics to distinguish non-AF rhythms. The whole sets of features are fed into a neural network classifier. Another approach uses the segments with 600 samples as the input of a 1 dimensional convolutional neural network. The output obtained from both approaches are combined using a decision table and finally the recordings are classified into three classes.

Results: The proposed method is evaluated using scoring function from 2017 PhysioNet/CinC Challenge and achieved an overall score of $80 \%$ and $71 \%$ on the training dataset and hidden test dataset, respectively.
\end{abstract}

\section{Introduction}

Electrocardiogram (ECG) is the most prevalent diagnostic tool for detecting cardiac arrhythmias. Any abnormal electrical activity of the heart can be detected using ECGs.

Atrial Fibrillation (AF) which occurs in $1-2 \%$ of the general population is one of the leading causes of stroke. Hence, early diagnosis will help to prevent major disasters [2]. Since it is caused by uncoordinated atrial activity, $\mathrm{P}$ wave does not exist in ECG. Another sign of AF is RR irregularity. Detecting AF is a challenging issue because it is asymptomatic [3] and also appears briefly in ECG. It is a more difficult task if ECG signals are contaminated with noise. Therefore, AF detection algorithms need more precise preprocessing steps including applying nonlinear filters. AF detection methods are based on two different approaches: atrial activity analysis based method [4] and ventricular response based method [5]. First one concentrates on absence of $\mathrm{P}$ wave or disorder in $\mathrm{P}$ wave morphology. $\mathrm{F}$ wave appearance in ECG baseline can also be an indication of AF. The other method investigates heart rate variability in signals by detecting QRS complex. This procedure is noise resistant because of high amplitude of $\mathrm{R}$ peaks.

The PhysioNet/CinC Challenge 2017 [1] aims to classify ECG signals into 4 classes of arrhythmias and develop an automated algorithm to assess AF.

The proposed paper describes the data set used in section 2.1. The preprocessing is discussed in section 2.2. Section 2.3 and section 2.4 explain the feature based approach and give details about the features in different domains. In classification section the two separate outlooks, deep learning and feature based approaches are presented. Finally, the obtained results and discussions are explained in Sections 4 and 5, respectively. 


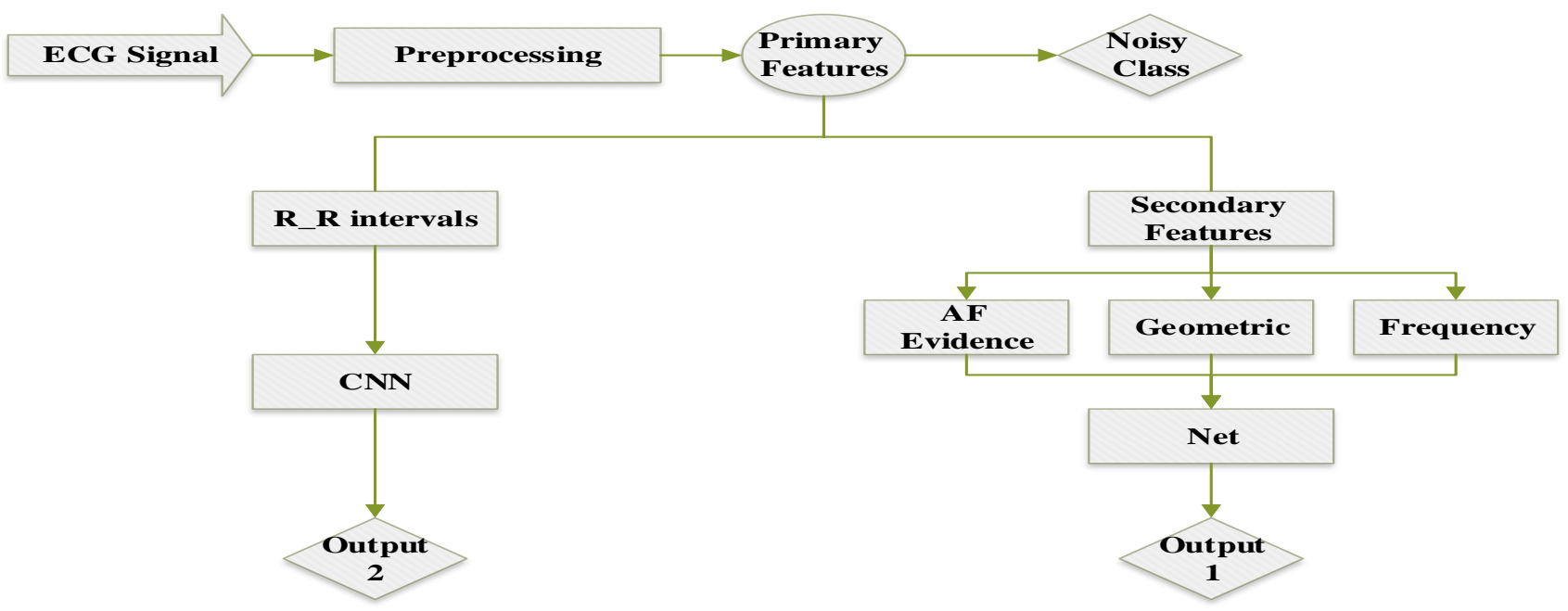

Figure 1. Block diagram of proposed method.

\section{Materials and Methods}

In this section the detailed steps of the algorithm are discussed. The complete flowchart of the proposed method is also shown in Figure 1.

\subsection{Dataset}

The atrial fibrillation detection database consists of 8528 ECG signals. Records are labelled as Noisy, Normal, AF and other rhythms. Sample frequency of recordings are 300 $\mathrm{Hz}$ and duration of them varies from 9 s to 30 s. Our method is evaluated on a separated validation set of 300 ECG recordings which is provided by PhysioNet/CinC challenge 2017 for training set. More information can be found in [1].

\subsection{Preprocessing}

Baseline wandering removal and Butterworth low and high pass filter are first applied to all signals. Then, the fiducal points of ECG signals (P, QRS complex and T) are detected using the classical pan Tompkins algorithm [6]. Each heart beat is extracted using the detected R-peaks without the inclusion of the first and last beat. In order to ignore the cycles contaminated with noise and artifacts, cycle quality assessment is applied to each heart beat and the remained high quality signals are used for further processing [7]. Finally, to address the problem of amplitude scaling and eliminate the offset effect, the remained signal is normalized with Z-score normalization

\subsection{Morphological features}

In case of detecting abnormal heart rhythms especially $\mathrm{AF}$ and the irregularity of heartbeats, the dynamics are more relevant in the geometrical features of signals. Therefore, 4 features related to morphological characteristics of ECG signals are extracted as follows:

1. Geometric

After detecting the exact onset locations of $\mathrm{T}$ and $\mathrm{Q}$ points, T-Q segment is separated for each heart cycle. The ratio of the circumference of signal with respect to the total duration of T-Q segment is used as the first feature.

2. Fractal dimension

This feature which is related to complexity of dynamic of a system represents the self-similarity of signals [8].

\section{Correlation coefficient}

This feature is a quantitative measurement of dependency of two time series. In our case, each two adjacent cycles are correlated and the average number of all correlation coefficients is used as a feature for each signal.

4. Variance of $R$ peaks

The standard deviation of the $\mathrm{R}$ points in each signal can be a good detector of irregularity of signal.

\subsection{Time and frequency domain features}

Alongside morphology features, time and frequency domain features can also be good discriminator of abnormal heart rhythms. 3 features are used in this section. The first feature is the histogram of RR intervals that can represent the heart rate variability (HRV) of signals. Moreover, there are useful information hidden in the frequency spectrum of signals. Thus, the power ratio of the frequency band $5-10 \mathrm{~Hz}$ to the total power of each TQ interval is also used as another feature. One of the main 
properties of abnormal heart rhythms other than AF, is the existence of premature beats. These beats often exist two or three times in signals and they mostly have a negative polarity. Sudden change of amplitude and polarity of premature beats are used to detect them. Besides, heart rates lower than $45 \mathrm{bpm}$ or higher than $97 \mathrm{bpm}$ are assumed as other arrhythmias.

\subsection{Classification}

Two different approaches are used for the classification. The first approach uses the features explained in previous sections and the second one employs deep neural networks. The final output is labeled to each recording using a decision table.

1. Feature based classification:

Fractal dimension, correlation coefficient and variance of $R$ peaks noted as primary features in the flowchart are used to detect noisy signals at first by comparing each feature to an optimum threshold. The remaining features which are denoted as secondary features are fed into a neural network classifier to predict normal, AF and signals related to other arrhythmias.

2. Deep neural networks

In this section after the preprocessing is done for each recording the signals are divided to segments as an input of a 1 dimensional deep learning convolutional neural network $(\mathrm{CNN})$. These segments consist of 600 samples (350 samples before R-peaks and 250 samples after Rpeaks). Table 1 demonstrates the detailed structure of the $\mathrm{CNN}$ and each part is discussed.

Architecture: The standard architecture of a CNN consists of four components:

- Convolution layer

The convolution layer is the main building block of a CNN. This layer does most of the computational intensive lifting. The prime objective of convolution is to extract features from the input ECG signals. The convolution layers are arranged in feature maps [9] (11 layers of feature maps in total).

- Activation function

In general, activation function serves to map nonlinearity into the data [9]. In this work, the Exponential linear unit (ELU) [10] is used as an activation function for layers 1, 3, $5,7,9$, and 10. Also, the softmax function is implemented for layer 11 (last layer).

- Pooling layer

Pooling also referred to as downsampling, is an operation to condense features and computational complexity of the network. The max-pooling operation is employed in this work. Max-pooling outputs only the maximum number in each kernel, thus reducing the feature map size [9].

Kernel size also refers to the size of the filter which convolves around the feature map while stride controls how the filter convolve around the feature map [9]. The amount by which the filter slides is the stride. In this work, the stride is set at 1 . Therefore, the filter convolves around the different layers of feature map by sliding one unit each time.

- Fully connected layer

The final layer of the fully-connected network is a softmax layer with an output of $X$ dimensional vector where $X$ is the number of classes that we desire to have. In this study, it is a three-class (Normal, AF and Other arrhythmias) problem, hence, $\mathrm{X}$ is set at 3 in this work.

Table 1. The details of CNN structure are shown.

\begin{tabular}{|c|c|c|c|c|c|}
\hline layer & Type & $\begin{array}{c}\text { Neuron } \\
\text { size }\end{array}$ & $\begin{array}{c}\text { Kernel } \\
\text { Size }\end{array}$ & $\begin{array}{c}\text { Filter } \\
\text { Numb } \\
\text { er }\end{array}$ & $\begin{array}{l}\mathrm{St} \\
\text { ri } \\
\mathrm{de}\end{array}$ \\
\hline $0-1$ & Convolution & $574 \times 16$ & 27 & 16 & 1 \\
\hline $1-2$ & MaxPooling & $287 \times 16$ & 2 & - & 2 \\
\hline $2-3$ & Convolution & $274 \times 32$ & 14 & 32 & 1 \\
\hline $3-4$ & MaxPooling & $\begin{array}{l}137 \times \\
32\end{array}$ & 2 & - & 2 \\
\hline $4-5$ & Convolution & $\begin{array}{l}134 \times \\
32\end{array}$ & 4 & 32 & 1 \\
\hline $5-6$ & Maxpooling & $67 \times 32$ & 2 & - & 2 \\
\hline $6-7$ & Convolution & $65 \times 10$ & 3 & 10 & 1 \\
\hline $7-8$ & MaxPooling & $33 \times 10$ & 2 & - & 2 \\
\hline $8-9$ & $\begin{array}{l}\text { FullyConnec } \\
\text { ted }\end{array}$ & 180 & - & - & - \\
\hline $9-10$ & $\begin{array}{l}\text { FullyConnec } \\
\text { ted }\end{array}$ & 42 & - & - & - \\
\hline $10-11$ & Softmax & 3 & - & - & - \\
\hline
\end{tabular}

Training: Adam optimizer [11] with a batch size of 16 is executed in this work. The dropout and initial learning rate parameters are set to 0.75 and 0.001 respectively. These parameters are tuned accordingly to obtain optimum performance. Also, Xavier initialization method is used for weights initialization.

\section{Results}

The outputs of both classifiers discussed in the previous section are combined using a decision table. (Table 3) Output 1 and output 2 refer to the prediction of neural network and $\mathrm{CNN}$ respectively.

Our algorithm is assessed on both the training and hidden test set provided by Physionet/CinC challenge 2017. The scores obtained from training and test set are demonstrated in Table 4, respectively. 
Table 3. Decision table.

\begin{tabular}{c|c|ccc}
\hline \multirow{4}{*}{ Output 2 } & \multirow{3}{*}{ Result } & AF & Normal & Other \\
\cline { 2 - 5 } & AF & AF & AF & AF \\
& Normal & AF & Normal & Normal \\
& Other & AF & Other & Other \\
\hline
\end{tabular}

Table 4. Evaluation of the proposed method on validation and hidden test set

\begin{tabular}{cccc}
\hline & classes & $\begin{array}{c}\text { Training } \\
\text { scores }\end{array}$ & $\begin{array}{c}\text { Hidden } \\
\text { test } \\
\text { scores }\end{array}$ \\
\hline \multirow{4}{*}{ Prediction } & Normal & 0.8804 & 0.8664 \\
& AF & 0.7881 & 0.7179 \\
& Other & 0.67 & 0.5463 \\
& overall & 0.78 & 0.71 \\
\hline
\end{tabular}

\section{Discussion}

This research aims to distinguish different kinds of arrhythmias form normal and noisy signals.

For the noisy class, since the cycles have no similarity the correlation coefficient of successive inter-beats and fractal dimension can detect them with high accuracy. Standard deviation of $\mathrm{R}$ peak amplitudes are also calculated and compared to optimum threshold. Noisy signals have larger standard deviation of $\mathrm{R}$ peaks.

The main focus is to identify indicators which are designated to AF class. Although HRV can be a good indicator to classify normal and $\mathrm{AF}$ recordings but it is not a good feature to distinguish AF from other rhythms. Because most of abnormal heart rhythms are indicated by HRV. Therefore, besides the feature related to histogram of RR interval another sets of features should be combined to discriminate $\mathrm{AF}$ form other classes. From a morphological point of view, AF recordings lack the existence of $\mathrm{P}$ wave. This indicator is well identified by the geometric feature. Another indicator of AF arrhythmia is the existence of $f$ waves in the TQ interval of a heart cycle. These waves cause signals to have more power in the frequency range of $5-10 \mathrm{~Hz}$ compared to normal or other arrhythmias.

We implemented both the feature based approach and deep learning approach to understand which of them can identify each class.

From the results obtained separated for each approach, it is understood that the first feature based approach is good at detecting the $\mathrm{AF}$ class form other and normal class and the trained $\mathrm{CNN}$ can better distinguish other class from $\mathrm{AF}$ and normal. This is the logic behind the decision table of how to combine the output of both classifiers.

It is suggested to consider other segments of signals for the input of the $\mathrm{CNN}$ and find optimal parameters for the structure.

\section{References}

[1] Gari Clifford, Chengyu Liu, Benjamin Moody, Ikaro Silva, Qiao Li, Alistair Johnson, Roger Mark. AF Classification from a Short Single Lead ECG Recording: the PhysioNet Computing in Cardiology Challenge 2017. Computing in Cardiology (Rennes: IEEE), Vol 44, 2017 (In Press).

[2] Andrade J, Khairy P, Dobrev D, Nattel S. The clinical profile and pathophysiology of atrial fibrillation. Circulation research. 2014 Apr 25;114(9):1453-68...

[3] Savelieva I, Camm A. Silent atrial fibrillation-another Pandora's box. Pacing and Clinical Electrophysiology. 2000 Feb 1;23(2):145-8.

[4] Ladavich S, Ghoraani B. Rate-independent detection of atrial fibrillation by statistical modeling of atrial activity. Biomedical Signal Processing and Control. 2015 Apr 30;18:274-81.

[5] Oster J, Clifford GD. Impact of the presence of noise on RR interval-based atrial fibrillation detection. Journal of electrocardiology. 2015 Dec 31;48(6):947-51.

[6] Mollakazemi MJ, Asadi F, Ghiasi S, Sadati SH. Applying quality index criterion for flexible multi-detection of heartbeat using features of multimodal data. InComputing in Cardiology Conference (CinC), 20162016 Sep 11 (pp. 10651068). IEEE.

[7] Abdollahpur M, Ghaffari A, Ghiasi S, Mollakazemi MJ. Detection of pathological heart sounds. Physiological measurement. 2017 Jun 8.

[8] Raghavendra BS, Dutt ND. Computing fractal dimension of signals using multiresolution box-counting method. International Journal of Information and Mathematical Sciences. 2010;6(1):50-65.

[9] LeCun Y, Bengio Y, Hinton G. Deep learning. Nature. 2015 May 28;521(7553):436-44.

[10] Clevert DA, Unterthiner T, Hochreiter S. Fast and accurate deep network learning by exponential linear units (elus). arXiv preprint arXiv:1511.07289. 2015 Nov 23.

[11] Kingma D, Ba J. Adam: A method for stochastic optimization. arXiv preprint arXiv:1412.6980. 2014 Dec 22.

Address for correspondence

Shadi Ghiasi

Mechanical engineering department, K.N.Toosi University of Technology, Tehran, Iran

Shadi1337@gmail.com 